\title{
Computing gold cluster energies with density functional theory: the importance of correlation
}

\author{
Piero Ferrari \\ Quantum Solid State Physics, Department of Physics and Astronomy, \\ KU Leuven, 3001 Leuven, Belgium \\ pieroantonio.ferrariramirez@kuleuven.be \\ Klavs Hansen \\ Center for Joint Quantum Studies and Department of Physics, School of Science, \\ Tianjin University, \\ 92 Weijin Road, Tianjin 300072, China \\ KlavsHansen@tju.edu.cn \\ June 17, 2021
}

\begin{abstract}
Energies calculated with density functional theory depend critically on the choice of the exchange-correlation functional. In this work, we use measured dissociation energies of $\mathrm{Au}_{n}^{+}(n=5-17)$ clusters as benchmark data to test two very different functionals for calculating total energies in these clusters; the simpler (and fast) PBE and the evolved (and expensive) B2PLYP double-hybrid functionals. PBE consistently gives poor agreement with the experimental results. In contrast, the B2PLYP functional, which implicitly includes electron correlation by performing a perturbative second-order correction, significantly improves the agreement of the calculations, at the cost of much more demanding computations. The better performance of the double-hybrid functional is ascribed to the longer range of the interatomic potential.
\end{abstract}




\section{Introduction}

The properties of gold clusters have attracted significant interest after the discovery that these particles are catalytically active ${ }^{1}$, in sharp contrast to the bulk material. Presently, a number of properties of Au clusters have been studied, including thermodynamic stabilities $^{2}$, optical responses ${ }^{3}$, reactivities ${ }^{4}$, and de-excitation channels ${ }^{5}$, as well as geometric structures ${ }^{6}$ and ground state energies ${ }^{7}$. In parallel to experimental studies, quantum chemical methods have been a very important tool for understanding the properties of Au clusters. They have allowed an identification of stable geometries, the calculation of electronic structures and the computation of energetics (and thus all related properties, such as ionization potentials and binding energies). In this connection, density functional theory (DFT) has been the method of choice in most studies dealing with gold clusters, given its decent accuracy and low computational cost. Nowadays, gold clusters of even large sizes can be investigated computationally using this method $^{8}$.

At least for small clusters, structures can be optimized by a number of different procedures, ranging from simple steepest descent to different biased sampling methods ${ }^{9}$. Although the outcome of a geometric optimization may be fairly reliable, modulo the influence of the precise energy functional on the exact geometries, energies may still be calculated incorrectly to a significant degree. It should be noted that most effects studied experimentally are energetic in nature, and that a sufficiently good geometric optimization is no guarantee that such energetic quantities are predicted correctly. Problems associated with energy functionals of other materials are accentuated by the well known difficulties for gold caused by relativistic effects ${ }^{10}$.

The situation is perhaps most clearly illustrated by the fact that in diverse studies, the structures of gold clusters calculated by DFT agree very well with experimental data, as in ion mobility ${ }^{11 ; 12}$, electron diffraction ${ }^{13}$, photoelectron spectroscopy ${ }^{14}$ and infrared multiple photon dissociation spectroscopy ${ }^{6 ; 15}$ experiments. The binding energies, however, show serious discrepancies to the experimentally measured values, available for $\mathrm{Au}_{n}^{+}$up to $n=27^{7}$. Commonly, benchmark analyses are performed for the smaller clusters, such as an Au dimer, and based on this analysis, conclusions as to which theory level should be employed are extrapolated to larger sizes ${ }^{16}$. A satisfactory numerical result in the small dimeric system, however, does not guarantee a good description for larger clusters. 
As the experimental data in Ref. ${ }^{7}$ can be considered benchmark results in a broad size range, it is worth reviewing briefly the methods by which they were obtained. The energies measured in the experiments were the evaporative activation energies ${ }^{17}$. The experiments were performed on cationic gold clusters in a Penning trap, applying single photon absorption via laser light to induce unimolecular decay ${ }^{7 ; 18}$. Several different experimental protocols were used to extract the dissociation energies. In the simplest version of the method, the unimolecular decay rates were measured time-resolved as a function of photon energies for two consecutive cluster sizes, for cluster size $n$ decaying into size $n-1$ and for $n+1$ decaying into $n-1$ via $n$. When the rate constants of the last decay in these two processes, the decay $n$ to $n-1$, are identical the difference in photon energies absorbed by $n+1$ and by $n$ gives the dissociation energy of cluster size $n$. The photon energy needs to be corrected by the small kinetic energy release, but this can be estimated fairly reliably. Other variations of the method were tested on the same clusters, for example using the energy dependent dimer-monomer branching ratio, which is then used as the calorimeter process instead of the rate constant. It should be stressed that the values bypasses all discussion of the correct modeling of the rate constants of the evaporation processes because these are not used, and that the method also works in the presence of a competing channel, such as radiative cooling ${ }^{19}$. This is of particular relevance for gold, for which the very strong recurrent fluorescence radiation present in these clusters ${ }^{5}$ will prevent a traditional analysis in terms of a pseudo-Arrhenius analysis. The agreement of the mass spectra first observed in the pioneering work of Katakuse $^{20}$ and the abundances calculated with the dissociation energies in ${ }^{7}$ provides a satisfactory confirmation of these experimentally determined model-free values (see Ref. ${ }^{21}$ for the comparison).

For completeness it should be mentioned that in principle, the differences in ground state energies of two clusters do not necessarily give their evaporative activation energies. Such an identification requires that there is no activation energy for attachment. Such reverse activation energies are fairly infrequent and have not been reported for measurements on metallic clusters, or in fact for any clusters at all, to the best of our knowledge. Even the well studied fullerenes which must be characterized as molecules and should be prime candidates to show such an attachment barrier do not show any sign of such a barrier for adsorption of both $\mathrm{C}$ and $\mathrm{C}_{2}$, down to limits of, conservatively estimated, several tens of $\mathrm{meV}$. The behavior of atomic clusters in this respect agrees with the bulk behavior of almost all elements, with zinc as the only known exception. 
A more direct indication for the gold clusters is a comparison of the measured activation energies for monomer and dimer loss. The dissociation energies for two sequential monomer losses add up to the dissociation energy for separating out a dimer and the dissociation energy of the dimer itself. In the presence of reverse activation energies, this does not hold for the measured evaporative activation energies, except by an accidental cancellation. The four cluster sizes for which this comparison can be made $(n=9,11,13,15)$ show good agreement with the discrepancies of $0.2,0.9$, 0.2 , and $0.2 \mathrm{eV}$, with only the high value being marginally significant. To place these differences in a proper context, a comparison with the much larger differences in dissociation energies found below for the two different functionals used here is relevant (see below). In summary, we can identify dissociation energies from the measured activation energies with confidence, which will be done in the following, and these model-free dissociation energies can be taken as benchmark values against which the performance of computational schemes should be judged.

A brief comparison of theoretical values was already given in Ref. ${ }^{7}$, mainly listing the values of dissociation energies vs cluster size that had been published by different workers. Some values had to be extracted from results on neutral clusters, with the use of experimentally measured ionization energies. Although such a procedure will potentially introduce errors due to the difference between vertical and adiabatic values, this difference did not seem to play any role when comparing final values for neutral and charged clusters. Here, however, we have made the conservative choice of comparing only with values calculated directly for the cations. We will show that selecting an appropriate theory level is crucial for correctly extracting energy-related values for the clusters.

\section{Computational details}

The density functional theory calculations were performed using the ORCA 4.2.1 software package ${ }^{22}$. Two functionals were selected for the analysis; the popular and computationally cheap (GGA) PBE functional ${ }^{23}$, and the advanced, but computationally demanding, B2PLYP ${ }^{24}$. The latter functional belongs to the double-hybrid category, in which a fraction of exact exchange from HartreeFock is mixed up with a fraction of exact correlation, by performing a perturbative second-order (PT2) correction. While

double-hybrids are found at the top of Perdews Jacobs ladder ${ }^{25}$ and are thought to 
provide the most accurate energy calculations within the DFT formalism, they are computationally very demanding, due to the PT2 step. Recently, the B2PLYP functional was shown to correctly describe the interaction of $\mathrm{Au}^{+}$ions with $\mathrm{Ar}$ atoms by a comparison of calculations of vibrational modes with measured infrared spectra ${ }^{26}$. In addition, an extensive benchmark analysis has shown that double-hybrids achieve an outstanding performance when computing electronic excited states in molecules ${ }^{27}$. Moreover, calculations on the neutral $\mathrm{Au}_{8}$ clusters, focused on the energy competition between planar and three-dimensional isomers, showed good agreement between results employing B2PLYP and those from coupled-cluster $(\operatorname{CCSD}(\mathrm{T}))$ theory ${ }^{28}$. In the work of Ref. ${ }^{28}$, it was argued that such agreement is a consequence of the correct description of long-range interactions in double-hybrids, due to the PT2 term. Such long-range correlation is known to be poorly described in conventional $\mathrm{DFT}^{29}$.

All calculations were performed by employing the Def2-TZVPP basis set ${ }^{30}$, with the inclusion of dispersion forces by applying the B3BJ correction ${ }^{31}$. In addition, the Stuttgart Def2-ECP pseudopotentials for Au were used, treating explicitly 19 valence electrons per Au atom ${ }^{30}$. Scalar relativistic effects are included in the ECP. In addition to the DFT calculations, and only for the neutral dimer $\mathrm{Au}_{2}$, coupled-cluster $\operatorname{CCSD}(\mathrm{T})$ calculations were performed, in conjunction with the Def2-TZVPP basis set and the Def2-ECP pseudopotentials. For $\mathrm{Au}_{2}$, the potential energy surface (PES) along the internuclear distance $(r)$ was constructed, using the B2PLYP and PBE functionals, in addition to $\operatorname{CCSD}(\mathrm{T})$. The curves were calculated up to $r=14 \AA$.

The lowest-energy structures of $\mathrm{Au}_{n}^{+}$clusters have been determined recently for the $n \leq 9$ size range by a combination of infrared multiple photon dissociation spectroscopy and DFT calculations ${ }^{15}$. Here we adopt those geometries for the calculations. In the work of Ref. ${ }^{15}$, the co-existence of a single three-dimensional and two planar isomers of $\mathrm{Au}_{8}^{+}$was confirmed in molecular beams at finite temperatures, with a much higher population of the three-dimensional geometry. We have therefore adopted that isomer for our calculations. The three isomers, however, are very close in energy (within 0.1 $\mathrm{eV}$ ) and as we deal with total energies, conclusions here are unaffected by this choice. In the $n=10-17$ size range, an extensive structural search was conducted in Ref. ${ }^{32}$, where an agreement was found for the predicted lowest-energy structures up to $n=13$ with those determined by ion mobility ${ }^{11}$. For this work, we have adopted the lowestenergy structures found in Ref. ${ }^{32}$. The adopted $\mathrm{Au}_{n}^{+}$structures are presented in Fig. 1, after re-optimization at the B2PLYP level. Based on those geometries, dissociation 


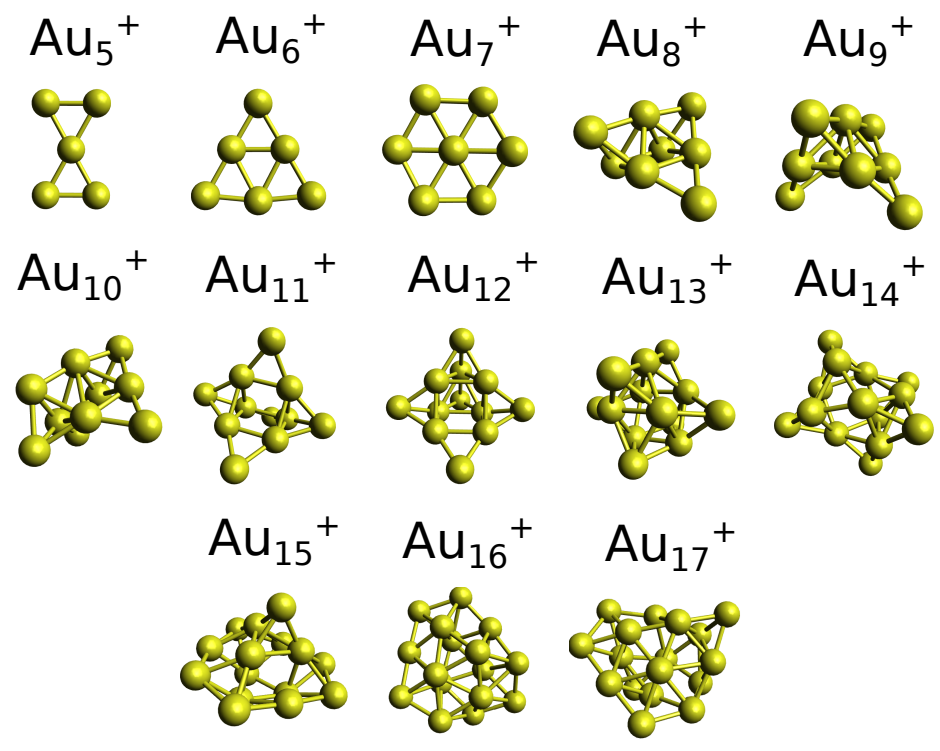

Figure 1: The structures of $\mathrm{Au}_{n}^{+}$clusters, optimized with the B2PLYP funcitonal. The geometries in the $n=5-9$ and the $n=10-17$ size range are adopted from Ref. ${ }^{15}$ and Ref. ${ }^{32}$, respectively, and relaxed as described in the main text.

energies of monomer decay were calculated, defined as:

$$
D_{n}^{m}=E\left(\mathrm{Au}_{n}^{+}\right)-E\left(\mathrm{Au}_{n-1}^{+}\right)-E(\mathrm{Au})
$$

\section{Comparison of dissociation energies}

Fig. 2 presents the monomer loss dissociation energies calculated for $\mathrm{Au}_{n}^{+}$clusters in the $n=7-17$ size range, computed with the B2PLYP and PBE functionals. In addition to the theoretical values, the experimentally determined dissociation energies, extracted from Ref. ${ }^{7}$, are shown. The experimental values show a pronounced odd-even pattern, with higher values for the odd- $n$ clusters. This effect is well-known for monovalent metal clusters, such as gold, and reflect the higher relative stability of clusters having paired spins ${ }^{33 ; 34}$. Here, the high stabilities occur for odd- $n$ clusters, given the cationic state of the system.

The dissociation energies calculated with the PBE functional also show the oddeven pattern, however, with values that are systematically lower than the experimental results. The disagreement becomes increasingly severe from $n=12$ and up, where the 


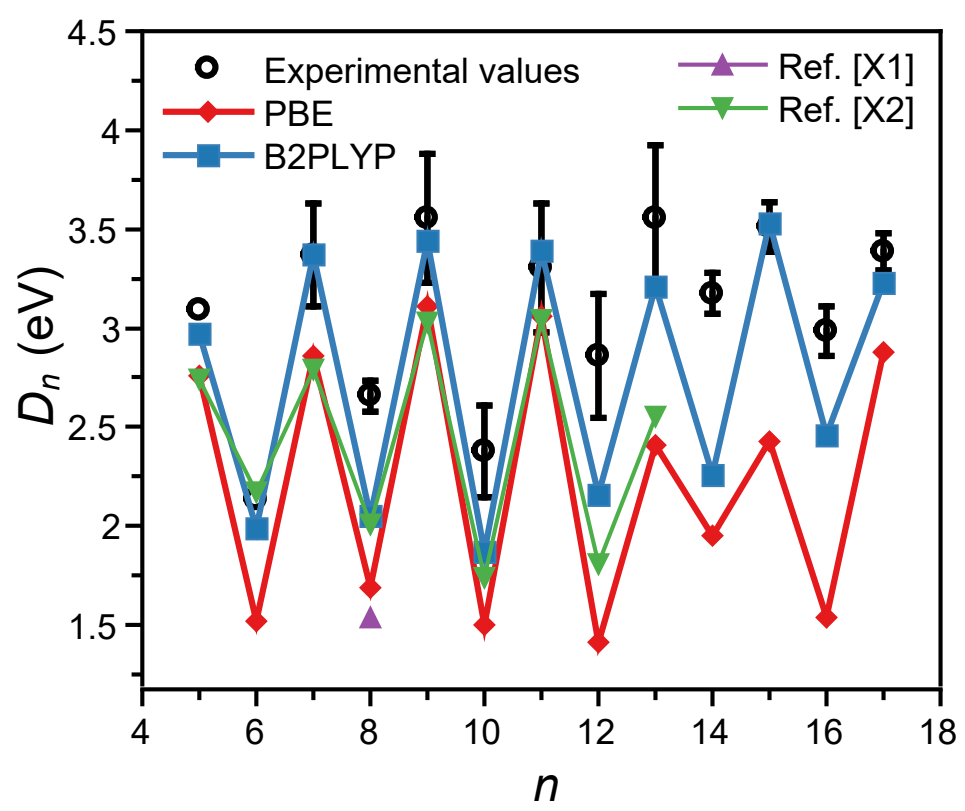

Figure 2: Comparison of theoretical dissociation energies with the experimentally determined values (reproduced from Ref. ${ }^{7}$ ). The calculations performed with the PBE functional are shown as diamonds and with the B2PLYP functional as squares.

calculations are more than $1 \mathrm{eV}$ off. Except for $\mathrm{Au}_{11}^{+}$, all the dissociation energies calculated with PBE are outside the error margin of the experiment. Moreover, also the relative values of dissociation energies are poorly reproduced by this level of theory; for example, the calculated values for $\mathrm{Au}_{13}^{+}$and $\mathrm{Au}_{15}^{+}$are much lower than those of $\mathrm{Au}_{11}^{+}$ and $\mathrm{Au}_{17}^{+}$, whereas the experimental numbers are quite similar.

The values calculated with the B2PLYP functional, in contrast, are much closer to the experimental results. For example, this method yields dissociation energies that are within the experimental uncertainty for $n=5,6,7,9,11,13$ and 15 . In general, the dissociation energies calculated for all even electron number clusters are in very good agreement with the experiment. For the odd electron number clusters an undershoot is clearly present which nevertheless still leaves them with values that are always closer to the experimental values than those calculated with the PBE functional. The price paid for this improved accuracy is a simulations with the B2PLYP functional which takes five times longer than with $\mathrm{PBE}$, and also requiring much higher disk memory.

A comparison with computed dissociation energies from the literature is also of interest. In Ref. ${ }^{11}$, the (GGA) BP86 functional was employed in conjunction with the 


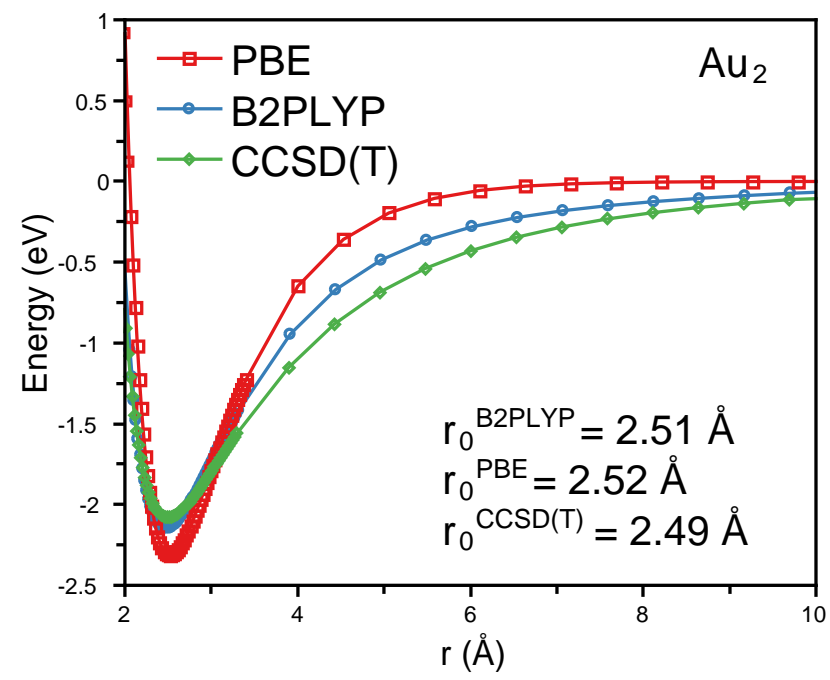

Figure 3: Potential energy surface (PES) curves of $\mathrm{Au}_{2}$, calculated with the PBE and B2PLYP functionals, as well as by $\operatorname{CCSD}(\mathrm{T})$. The equilibrium bond lengths in the inset refer to the minima of the curves, i.e. disregarding the nuclear vibrational motion.

small Def2-SVP basis set, in order to calculate dissociation energies of $\mathrm{Au}_{n}^{+}(n<14)$ clusters. The results are shown as triangles pointing down in Fig. 2. With the exception of $\mathrm{Au}_{6}^{+}$, the values are always lower than our results with the B2PLYP functional, with particularly lower values for the larger calculated sizes, $\mathrm{Au}_{12}^{+}$and $\mathrm{Au}_{13}^{+}$. Moreover, in Ref. ${ }^{35}$, the hybrid B3LYP functional was used together with the LANL2DZ basis set in a calculation of the monomer dissociation energy of $\mathrm{Au}_{8}^{+}$, yielding again a value much lower than both our result with B2PLYP and the experimental value (shown as the triangle pointing up in Fig. 2). Therefore, to the best of our knowledge, our calculations employing the double hybrid B2PLYP functional are, overall, the best theoretical results available to date on these systems.

\section{Analysis}

In order to elucidate the reasons why B2PLYP performs better than other functionals, we have analyzed in more detail the geometries and the electronic structure of some selected cluster sizes, obtained with the B2PLYP and PBE functionals.

Our first analysis concerns a scan of the potential energy surface (PES) of $\mathrm{Au}_{2}$, 
shown in Fig. 3. For such a small system, the DFT calculations can also be compared with accurate $\operatorname{CCSD}(\mathrm{T})$ computations, which has a computational cost that scales with the size of the system as $N^{7}$ and therefore is non-viable for larger clusters. The equilibrium bond distance $\left(r_{0}\right)$ calculated by PBE is $2.52 \AA$, whereas the experimental value is the lower value of $2.47 \AA^{36}$. As for the range of the PES, the one calculated by PBE predicts a short range of the potential which approaches zero with increasing separation faster than either of the two other. The surface calculated by $\operatorname{CCSD}(\mathrm{T})$, in contrast, predicts an equilibrium bond distance of $2.49 \AA$, i.e., closer to the experimental value, but on the other hand a range of the potential which is much longer than that computed by PBE, as clearly seen in the figure. The differences in the PES between $\mathrm{PBE}$ and $\operatorname{CCSD}(\mathrm{T})$ are consequences of the incorrect asymptotic behavior of the potential energy in $\mathrm{DFT}^{28}$. Also the PES calculated by B2PLYP decreases slower with $r$ than with $\mathrm{PBE}$ and is, gratifyingly, closer to the $\operatorname{CCSD}(\mathrm{T})$ curve, which has an even longer range. This better asymptotic behavior is shared with other systems calculated with double hybrid functionals and is attributed to their additional PT2 step $^{28}$. The predicted equilibrium bond distance with B2PLYP is $2.51 \AA$, and therefore slightly better than with PBE.

Both the $\mathrm{Au}-\mathrm{Au}$ bond lengths predicted by the two functionals and the different range of the potentials influence the optimized geometries and energies, although the geometric effect will usually not cause shifts of global minima to other isomeric states for small clusters. As an example of the shift in geometry, the optimized structures of $\mathrm{Au}_{7}^{+}, \mathrm{Au}_{8}^{+}$and $\mathrm{Au}_{13}^{+}$, at the B2PLYP and PBE levels of theory, are compared in Fig. 4. While the geometries calculated at the two levels appear identical at first sight, closer inspection of the $\mathrm{Au}-\mathrm{Au}$ distances reveals systematically shorter values when employing B2PLYP, as expected from the PES analysis in Fig. 3. The different ranges of the potentials will cause the two energies to differ also for close or even identical geometries. For larger clusters, the difference in the potentials will likely cause a reordering of the many isomeric PES minima one must expect to be present for such relatively short-range potentials. As an example of the influence of the functional on geometry, the energy ordering of the four lowest-energy isomers of the $\mathrm{Au}_{8}^{+}$cluster were calculated using the B2PLYP and the PBE functionals, with an energy ordering presented in Fig. 5. While in both cases the three-dimensional Iso1 is found as the minimum-energy configuration (as shown experimentally in Ref. ${ }^{15}$ ), the ordering of the two planar (Iso3 and Iso4) and another three-dimensional geometry (Iso2) change 

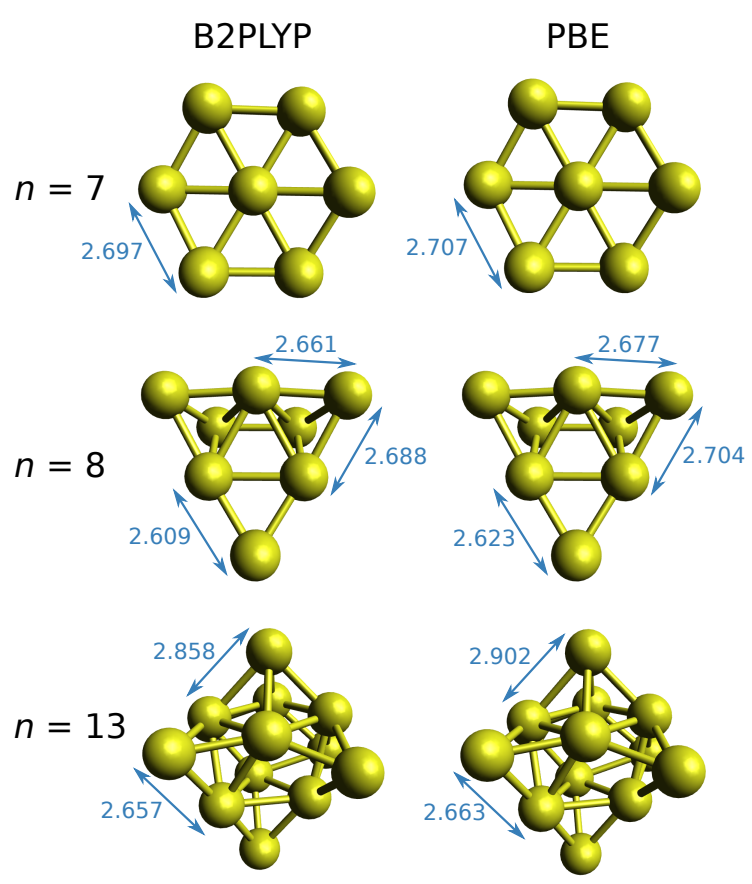

Figure 4: Geometry comparison for the $\mathrm{Au}_{7}^{+}$(top), $\mathrm{Au}_{8}^{+}$(bottom) and $\mathrm{Au}_{13}^{+}$(bottom) clusters, using the B2PLYP (left) and PBE (right) functionals. Bond lengths are given in units of $\AA$.

depending on the employed functional. In particular, Iso2 is largely stabilized with B2PLYP, being located $0.07 \mathrm{eV}$ above Ios1, whereas with PBE, Iso2 is found $0.27 \mathrm{eV}$ above Iso1.

It should be noted that although the bond lengths differ from the values for the dimer PES minima, the ratios are of similar magnitude, i.e. with slightly smaller values for the B2PLYP functional. The PES minimum of the gold dimer is higher for B2PLYP and based on equilibrium distances alone, the energies calculated with this functional would then be higher than calculated with the PBE function. This ordering is reversed by the longer range of the potential of the B2PLYP functional, which more than compensates it by enhancing binding by the next-nearest neighbors. This obviously schematic picture nevertheless rationalizes the observed behavior and is consistent with the expected higher performance of $\operatorname{CCSD}(\mathrm{T})$ that will push theory values even toward the experimental results. In summary, the difference in energy can therefore be understood as mainly a consequence of the difference range of the potential. 


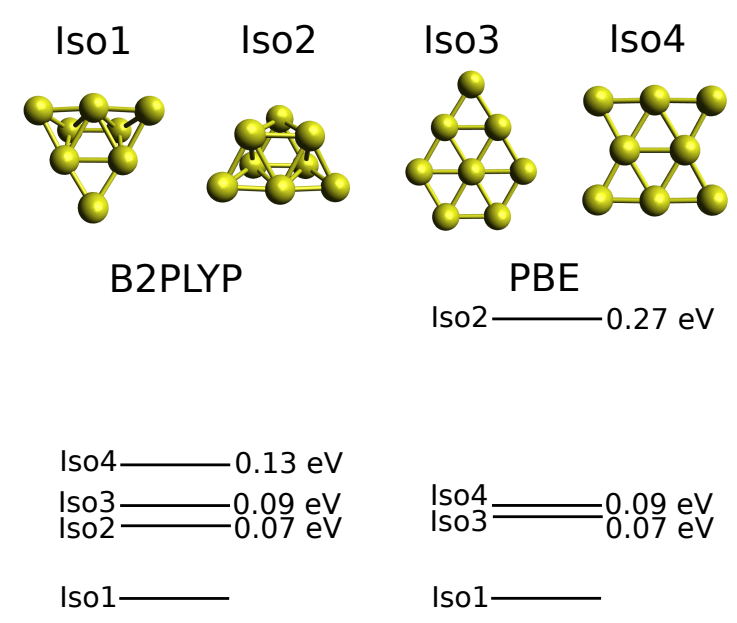

Figure 5: Energy ordering of the isomers of $\mathrm{Au}_{8}^{+}$, calculated using the B2PLYP and PBE functionals.

\section{Conclusions}

In this work, measured monomer dissociation energies of $\mathrm{Au}_{n}^{+}(n=5-17)$ clusters were used as benchmark data for testing total energies calculated with density functional theory. Two functionals of very different nature were employed for the analysis, the (GGA) PBE and the double-hybrid B2PLYP. The calculations employing the accurate and more computationally expensive B2PLYP functional yielded systematically better dissociation energies than PBE, over the entire investigated cluster size. The work demonstrates how important selecting a proper theory level is when quantifying physical property of gold clusters, and provide, to date, the most accurate calculations of monomer dissociation energies of these species.

\section{Acknowledgments}

P.F. is grateful to the FWO for a Senior Postdoctoral Fellowship. The resources and services used in this work were provided by the VSC (Flemish Supercomputer Center), funded by the FWO and the Flemish Government.

\section{Conflicts of interest}

There are no conflicts to declare. 


\section{References}

[1] M. Haruta, N. Yamada, T. Kobayashi and S. Iijima, Journal of Catalysis, 1989, 115, 301-309.

[2] N. Veldeman, E. Janssens, K. Hansen, J. De Haeck, R. E. Silverans and P. Lievens, Faraday Discussions, 2008, 138, 147-162.

[3] A. N. Gloess, H. Schneider, J. M. Weber and M. M. Kappes, J. Chem. Phys., 2008, 128, 114312.

[4] T. M. Bernhardt, International Journal of Mass Spectrometry, 2005, 243, 1-29.

[5] K. Hansen, P. Ferrari, E. Janssens and P. Lievens, Physical Review A, 2017, 96, 022511.

[6] P. Gruene, D. M. Rayner, B. Redlich, A. F. van der Meer, J. T. Lyon, G. Meijer and A. Fielicke, Science, 2008, 321, 674-676.

[7] K. Hansen, A. Herlert, L. Schweikhard and M. Vogel, Physical Review A, 2006, 73, 063202.

[8] P. V. Nhat, N. T. Si and M. T. Nguyen, The Journal of Physical Chemistry A, 2020, 124, 1289-1299.

[9] S. Heiles and R. L. Johnston, International Journal of Quantum Chemistry, 2013, 113, 2091-2109.

[10] P. Pyykko, Chemical Reviews, 1988, 88, 563-594.

[11] S. Gilb, P. Weis, F. Furche, R. Ahlrichs and M. M. Kappes, J. Chem. Phys., 2002, 116, 4094-4101.

[12] F. Furche, R. Ahlrichs, P. Weis, C. Jacob, S. Gilb, T. Bierweiler and M. M. Kappes, J. Chem. Phys., 2002, 117, 6982-6990.

[13] M. P. Johansson, A. Lechtken, D. Schooss, M. M. Kappes and F. Furche, Physical Review A, 2008, 77, 053202.

[14] S. Bulusu, X. Li, L.-S. Wang and X. C. Zeng, Proceedings of the National Academy of Sciences, 2006, 103, 8326-8330. 
[15] P. Ferrari, G.-L. Hou, O. V. Lushchikova, F. Calvo, J. M. Bakker and E. Janssens, Phys. Chem. Chem. Phys., 2020, 22, 11572-11577.

[16] C. J. Owen, N. R. Keyes, C. Xie, H. Guo and P. Armentrout, J. Chem. Phys., 2019, 150, 174305 .

[17] K. Hansen, Statistical Physics of Nanoparticles in the Gas Phase, Springer, 2018.

[18] M. Vogel, K. Hansen, A. Herlert and L. Schweikhard, Physical review letters, 2001, 87, 013401.

[19] P. Ferrari, E. Janssens, P. Lievens and K. Hansen, International Reviews in Physical Chemistry, 2019, 38, 405-440.

[20] I. Katakuse, T. Ichihara, Y. Fujita, T. Matsuo, T. Sakurai and H. Matsuda, International Journal of Mass Spectrometry and Ion Processes, 1985, 67, 229236.

[21] K. Hansen and P. Ferrari, Chinese J. Chem. Phys., 2019, 32, 167-174.

[22] F. Neese, WIREs Computational Molecular Science, 2018, 8, e1327.

[23] B. Hammer, L. B. Hansen and J. K. Nørskov, Phys. Rev. B, 1999, 59, 7413-7421.

[24] L. Goerigk and S. Grimme, WIREs Computational Molecular Science, 2014, 4, 576-600.

[25] J. P. Perdew, A. Ruzsinszky, J. Tao, V. N. Staroverov, G. E. Scuseria and G. I. Csonka, J. Chem. Phys., 2005, 123, 062201.

[26] L. Delgado-Callico, P. Ferrari, J. Bakker, F. Baletto and E. Janssens, Theoretical Chemistry Accounts, 2021, 140, 1-9.

[27] S. Grimme and F. Neese, J. Chem. Phys., 2007, 127, 154116.

[28] S. A. Serapian, M. J. Bearpark and F. Bresme, Nanoscale, 2013, 5, 6445-6457.

[29] O. A. Vydrov and G. E. Scuseria, J. Chem. Phys., 2006, 125, 234109.

[30] F. Weigend and R. Ahlrichs, Phys. Chem. Chem. Phys., 2005, 7, 3297-3305. 
[31] S. Grimme, S. Ehrlich and L. Goerigk, Journal of Computational Chemistry, 2011, 32, 1456-1465.

[32] P. Ferrari, H. A. Hussein, C. J. Heard, J. Vanbuel, R. L. Johnston, P. Lievens and E. Janssens, Phys. Rev. A, 2018, 97, 052508.

[33] H. Häkkinen, Advances in Physics: X, 2016, 1, 467-491.

[34] S. Neukermans, E. Janssens, H. Tanaka, R. Silverans and P. Lievens, Phys. Rev. Lett., 2003, 90, 033401.

[35] F. Remacle and E. Kryachko, J. Chem. Phys., 2005, 122, 044304.

[36] R. Wesendrup, T. Hunt and P. Schwerdtfeger, J. Chem. Phys., 2000, 112, 93569362. 